\title{
A Control-aware QoS Adaptation Co-design Method for Networked Control Systems
}

\author{
Octavian STEFAN, Toma-Leonida DRAGOMIR \\ Politehnica University Timisoara, \\ 2 Piata Victoriei, Timisoara, 300006, Romania \\ octavian.stefan@upt.ro,toma.dragomir@upt.ro
}

\begin{abstract}
The current study proposes a control-aware Quality of Service adaptation co-design method for networked control systems. The novel networked control structure is based on a remotely placed Quality of Service adapter that continuously changes the network parameters using the Next Steps in Signaling protocol suite for end-to-end Quality of Service. The control system's design is developed gradually and the system's stability is assessed by considering the overall system as a switched linear one. Finally, the results are validated on a numerical example.
\end{abstract}

Keywords: networked control systems, co-design control methods, switched linear systems.

\section{Introduction}

Advances in digital communication technologies offered new possibilities for the development of telecontrol applications. Consequently, networked control systems (NCS) have gained an increasing amount of attention in the scientific community. Although NCS have several advantages ([11]), there are some network induced issues like time-varying delays, data loss, and limited data transfer capacity which can affect the system's performance and stability ([24]). In order to overcome these issues, different control strategies are proposed in the specialized literature that can be divided in two categories: control over network solutions (e.g. gain scheduling ([15]), adaptive Smith predictor ([14]), optimal stochastic control ([18]), event based control ([12]), predictive control ([5]), communication disturbance observer ([17]) or robust control ([24])) and control of network solutions (e.g. end-to-end Quality of Service (QoS) ([8])). Although each category is proven to work in practice, best possible results are achieved when using co-design methods obtained by combining control solutions from both categories (best resource utilization) ([21]). Furthermore, when using a shared medium network to transport data for multiple NCS and other possible applications with an unknown traffic pattern, co-design methods are mandatory to assure the control objectives. According to [1], two types of co-design methods exist. The first one, referred to as "implementation-aware control law design", presumes real-time continuous adaptation of the control parameters according to the ones of the network (e.g. continuous adaptation of the sample rate ([2]) or continuous adaptation of the controller gains ([22]) based on the values of the network time delays and packet loss number). The second one, named "controlaware QoS adaptation" presumes a real-time reallocation of network resources, by modifying the QoS parameters, in order to maintain the quality of control (e.g. dynamic bandwidth allocation in a Switched Ethernet Network ([9])).

Current paper proposes a new control-aware QoS adaptation co-design method for NCS using the Next Steps in Signaling (NSIS) protocol suite for end-to-end QoS. In addressing the control objective (tracking and stabilization) a networked control structure is considered, composed out of a local plant, a remotely placed controller and a network adaptation block. Based on the network parameters' values, the network adaptation block performs a real-time continuous adaptation of the QoS parameters in order to assure the control objectives and to minimize the network resource utilization.

For the analysis stage, the study uses a switched linear system ([20]) as a network transmission model (NTM) - presented by the authors in [22] - that completely characterize network transmissions from an input-output perspective, taking into account time-varying delays, packet losses and irregular situations together with their handling strategy ([23]) that can occur when using unreliable networks and connectionless protocols. 
The remainder of this paper is organized as follows. Section 2 describes the problem formulation. Section 3 presents the NTM. Section 4 presents the design methodology for the control structure. Section 5 analyses the system's stability. Section 6 presents an illustrative example and Section 7 states some final conclusions.

\section{Problem Formulation}

Consider the NCS given in Figure 1. It corresponds to a control structure with two control loops: an internal loop driven through the control signal $\mathrm{u}_{\mathrm{f}}$, and an external loop driven through the control signal $u_{t}$. The external loop assures tracking features and a zero steady-state error due to the discretetime integrator.

The local side of the NCS consists of the controlled process, to which a state observer is attached, and the integrator element of the external loop. The observer was placed on local side to avoid the strong influence of the disturbances induced by network transmissions (e.g. time varying delays, packet loss ([11])). The increase of the computational burden is considered to be acceptable. Also, at the local side, the global control signal $u_{c}$ is computed from $u_{t, N}$ and $u_{f, N}$ (the received signals corresponding to $u_{t}$ and $u_{f}$ ).

The remote side of the NCS is composed of the controller, serving the control loops, and the QoS adapter which continuously adapts the network parameters by using information obtained from both paths of the network. The adapter manages both transmission channels (network paths) in order to maintain network transmission disturbances in certain limits imposed by the NCS design.

The aim of the current study is to design the controller and the QoS adapter for the NCS from Figure 1, capable of stabilizing the system and ensuring good tracking performances.

In order to analyse and design the control system implemented in Figure 1, five hypotheses are considered $\left(\mathrm{H}_{1}-\mathrm{H}_{4}\right.$ are adopted from [22]):

$\mathrm{H}_{1}$ : the process is a $\{\mathrm{u}\} \rightarrow\left\{\mathrm{y}_{\mathrm{p}}\right\}$ oriented SISO linear time-invariant system, controllable and observable;

$\mathrm{H}_{2}$ : the stabilization and output tracking of the control system can be achieved through state feedback integral control;

$\mathrm{H}_{3}$ : the network transmissions are characterized by time-varying delays, packet losses and irregular situations ([23]), and the network can be regarded as a switched linear system;

$\mathrm{H}_{4}$ : the tracking reference signal $\mathrm{w}$ is a stepwise signal, and can be modelled as the output of an exogenous first order reference generator;

$\mathrm{H}_{5}$ : from a systemic point of view, the QoS adapter represents an exogenous system that generates the switching signals of the network block bounded in certain limits.

Figure 2 illustrates the block diagram associated to the NCS in Figure 1 obtained like in [22] by taking into account the above hypothesis and the following remark:

$\mathrm{R}_{1}$ : a NCS with bidirectional network transmission paths (like the one in Figure 1) between a non-inertial controller and a timeinvariant controlled plant is equivalent - from the point of view of stability analysis - with one where the network is placed only on the feedback path (like in Figure 2) ([11]), ([6]).

The discreet-time networked control structure from Figure 2, working with the sample time h, is introduced for the study of the dynamical features of the NCS. The Network Channel's transmissions are affected, at every sample

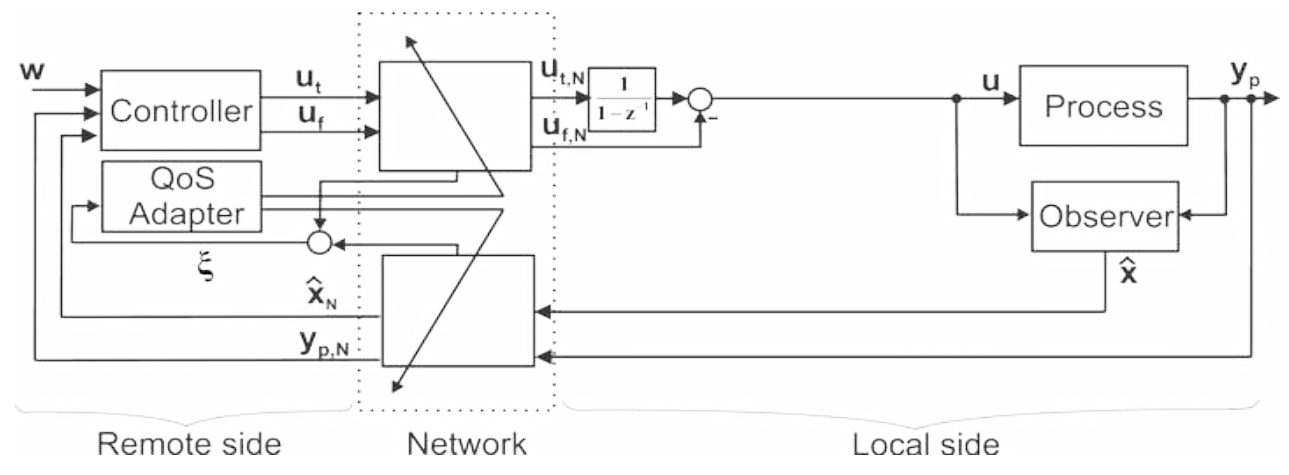

Figure 1. Proposed networked control structure 


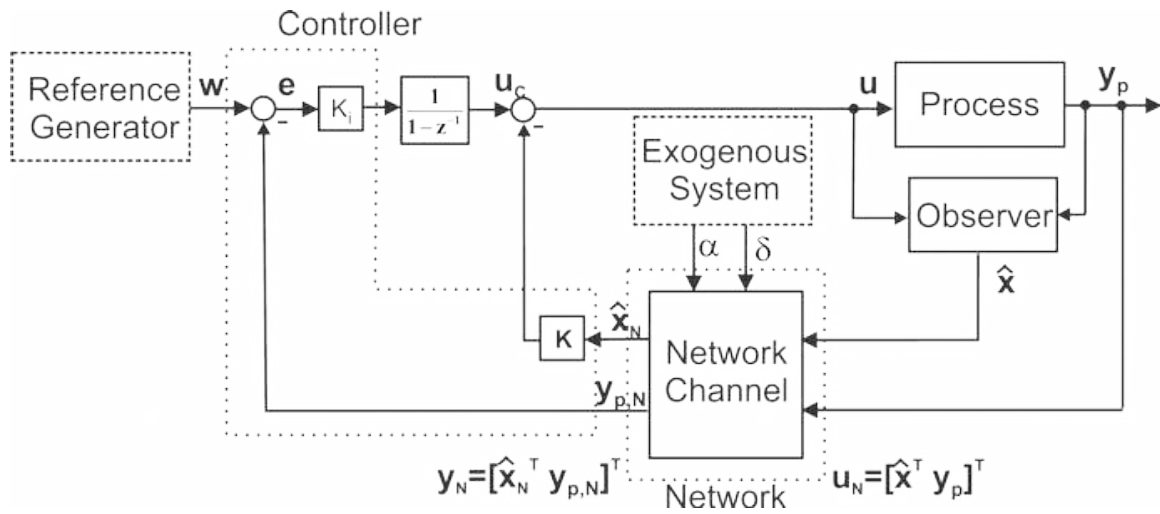

Figure 2. One-channel networked control system

instant, by the switching signals generated from the Exogenous System. Signal $\alpha$ contains information about the time delay $-\tau$ values (as multiples of the sample rate $h$ ) and packet loss flag $-\mathrm{p}$ values associated to the data packets transmissions, while $\delta$ selects the proper output values of the Network Channel.

\section{Network Model}

In order to analyse the NCS's behaviour, the switched linear system (1), derived in [22] from the nonlinear model proposed in [23], is considered as the model of the network:

$$
\left\{\begin{array}{l}
x_{N}[k+1]=\boldsymbol{A}_{\alpha[k], \delta[k]} \boldsymbol{x}_{N}[k]+\boldsymbol{B}_{\alpha[k]} \boldsymbol{u}_{N}[k] \\
y_{N}[k]=\mathbf{C} \mathbf{x}_{N}[k]
\end{array}\right.
$$

$\mathbf{u}_{N}, \mathbf{x}_{N}$ and $\mathbf{y}_{N}$ represent, respectively, the input, state and output variable of the network;

$\alpha: \mathbb{N} \rightarrow \Omega_{1}$ and $\delta: \mathbb{N} \rightarrow \Omega_{2}$ are switching signals, $\Omega_{1}$ is the set of possible values taken by $\tau[\mathrm{k}]$ at a given sample instant $\mathrm{k}:\{1,2,3, \ldots$, $\left.\tau_{\max }\right\}$, and $\Omega_{2}$ is the bivalent set: $\{0,1\}$;

$$
\begin{aligned}
& A_{\alpha[k], \delta[k]}=\left[\begin{array}{ll}
E_{x} I_{s r n} & 0_{r n \times n} \\
E_{a x} & E_{a}
\end{array}\right] \\
& B_{\alpha[k]}=\left[\begin{array}{l}
E_{u} \\
E_{a u}
\end{array}\right] E_{u \alpha} \\
& C=\left[\begin{array}{ll}
0_{n \times r n} & I_{n}
\end{array}\right] \\
& E_{x}=\sigma\left(\tau_{\max }-\alpha[k]-1\right) \cdot f_{r n}(\alpha[k]) \\
& +\left[1-\sigma\left(\tau_{\max }-\alpha[k]-1\right)\right] \cdot I_{r n} \\
& E_{a x}=\delta[k] \cdot\left[\begin{array}{lllll}
0_{n} & \sigma(\alpha[k]-2) & 0_{n} & \cdots & 0_{n}
\end{array}\right] \\
& E_{a}=\delta[k] \cdot \sigma(\alpha[k]-2) \cdot I_{n} \\
& E_{u}=\sigma\left(\tau_{\max }-\alpha[k]-1\right) \cdot I_{r n} \\
& E_{a u}=\sigma\left(\tau_{\max }-\alpha[k]-1\right) \cdot \sigma(1-\alpha[k]) \\
& \cdot\left[\begin{array}{lllll}
E_{u \alpha}^{1} & 0_{n} & 0_{n} & \cdots & 0_{n}
\end{array}\right]
\end{aligned}
$$

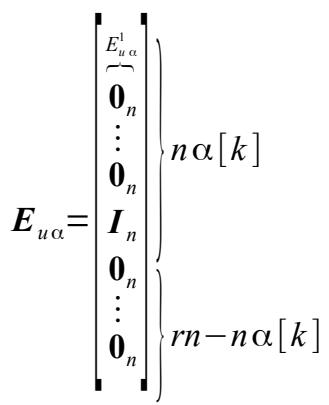

$$
\sigma(v)=\left\{\begin{array}{l}
0, v<0 \\
1, v \geq 0
\end{array}\right.
$$$$
\boldsymbol{I}_{s r n}=\left[\begin{array}{cccccc}
\mathbf{0}_{n} & \boldsymbol{I}_{n} & \mathbf{0}_{n} & \cdots & \cdots & \mathbf{0}_{n} \\
\vdots & \ddots & \ddots & \ddots & & \vdots \\
\vdots & & \ddots & \ddots & \ddots & \vdots \\
\vdots & & & \ddots & \ddots & \mathbf{0}_{n} \\
\vdots & & & & \ddots & \boldsymbol{I}_{n} \\
\mathbf{0}_{n} & \cdots & \cdots & \cdots & \cdots & \mathbf{0}_{n}
\end{array}\right]
$$

$$
\boldsymbol{f}_{r n}(\tau[k])=\operatorname{diag}(\sigma(\tau[k]-2), \ldots, \sigma(\tau[k]-r-1))
$$

$\mathbf{I}_{\mathrm{n}}$ and $\mathbf{I}_{\mathrm{rn}}$ are unitary matrices, $\mathbf{I}_{\mathrm{srn}}$ is defined as an $\mathbf{r} \cdot \mathrm{n}$ order quadratic matrix, $\sigma$ is the unitary matrix step function of scalar variable $\mathrm{v}$ and $\mathbf{0}_{\mathrm{n}}$ is the quadratic zero matrix of order $n$.

The model (1) characterizes network transmissions as signal time shifts and deformation processes (i.e. time delay and packet loss) and captures the irregular situations along with their associated handling strategy ([23]). It behaves as a buffer which is continuously shifted (passing of a new sampling instance), filled (new data packet arrival) and emptied (packet drop).

\section{Control Design}

The NCS in Figure 2 uses three discrete-time control elements, namely: the state integral controller, the state observer and QoS adapter, working with the sample time h. Consequently, 
the zero-order hold equivalent model of the SISO linear continuous-time invariant process is used for the process:

$$
\left\{\begin{array}{l}
x[k+1]=F_{d} x[k]+G_{d} u[k] \\
y_{p}[k]=H_{d}^{T} x[k]
\end{array},\right.
$$

where $\mathbf{x}$ is the state vector of dimension $n_{p}, u$ and $y$ are scalar input and output, $\mathbf{F}_{d}$ is a $n_{p} \times n_{p}$ matrix, $\mathbf{G}_{\mathrm{d}}$ and $\mathbf{H}_{\mathrm{d}}$ are $\mathrm{n}_{\mathrm{p}} \times 1$ vectors. According to the hypothesis $\mathrm{H}_{1},\left(\mathbf{F}_{\mathrm{d}}, \mathbf{G}_{\mathrm{d}}\right)$ is a controllable pair and $\left(\mathbf{F}_{\mathrm{d}}, \mathbf{H}_{\mathrm{d}}^{\mathrm{T}}\right)$ is an observable pair.

\subsection{Controller design}

The state feedback integral controller (see hypothesis $\mathrm{H}_{2}$ ), implementing the control law

$$
u(z)=K_{i} \cdot \frac{1}{1-z^{-1}} \cdot\left(w(z)-y_{p}(z)\right)-K \cdot x(z)
$$

is designed, as if it were directly coupled to the process (2). In (3) $u(z), x(z)$ and $w(z)$ are the $z-$ transforms of the discrete-time signals $\{\mathrm{u}[\mathrm{k}]\}$, $\{\mathbf{x}[\mathrm{k}]\}$ and $\{\mathrm{w}[\mathrm{k}]\}$. From the multiple design methods available to determine the compensators gains $\mathbf{K}$ and $\mathrm{K}_{\mathrm{i}}$, the classical pole allocation method is chosen.

\subsection{Observer design}

The state observer is considered to be of Luenberger type ([16]), having the model

$$
\left\{\begin{array}{c}
\hat{x}[k+1]=F_{d} \hat{x}[k]+G_{d} u[k] \\
\quad+L\left(y_{p}[k]-\hat{y}_{p}[k]\right) \\
\hat{y}_{p}[k]=H_{d}^{T} \hat{x}[k]
\end{array}\right.
$$

Here, $\hat{x}$ and $\hat{y}_{p}$ are of the same dimensions like $\mathbf{x}$, and $\mathrm{y}_{\mathrm{p}}$. The gain matrix $\mathbf{L}$, which adapts the observer to the process (2) is also designed through pole allocation.

\subsection{QoS adapter design}

When using multiservice networks to design NCS, network resource utilization becomes critical. In order to maintain the quality of control with minimum bandwidth allocation, the QoS adapter has to dynamically reserve network resources for control, based on the quality of network transmissions. The NSIS protocol suite provides a generic framework capable of QoS signaling, supporting both sender and receiver initiated reservations ([25]), making it suitable for either local or remote QoS adapter design. The adapter implements the QoS NSIS Signaling Layer Protocol (NSLP) for signaling QoS reservations. The protocol specifications do not define a specific mechanism for obtaining QoS, permitting the definition of different methods for QoS modelling depending on the network infrastructure ([13]). Figure 3 presents the NSIS-based QoS adaptation for the NCS proposed in Figure 1. The QoS NSLP messages are sent peer-to-peer through each network node both ways from the Initiator to the Receiver and backwards.

The NSIS based QoS adapter can implement several designs, based on different QoS models. Current paper proposes a finite-state machine design using the QoS model proposed in [4] for Ethernet networks which defines eight priority classes for network traffic.

It is important to mention that the networked control structure from Figure 2 can also be associated to the NSIS-based NCS from Figure 3 for the analysis stage.

\section{Stability Analysis}

In order to analyse the NCS's behaviour, the model of the closed loop from Figure 2 should be first assembled. Because all the external signals were assigned to exogenous systems, the NCS's model is autonomous. The model is obtained by coupling, according to the connexions in Figure 2, the process model (2), the model of the associated observer (4), the

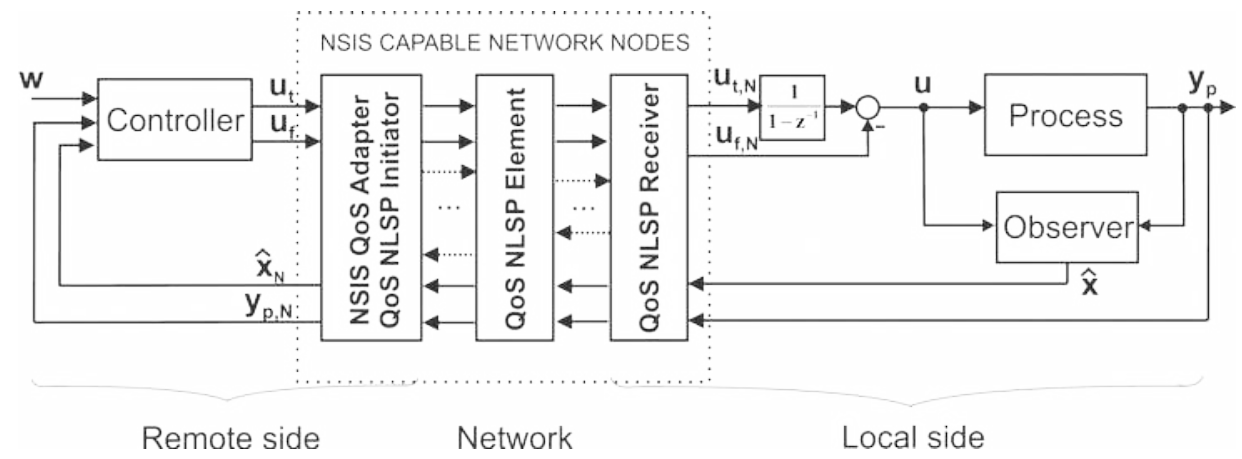

Figure 3. NSIS-based networked control system 
model of the feedback integral controller (3), and the network model (1).

Regarding the closed loop model's assembling, three remarks should be mentioned:

$\mathrm{R}_{2}$ : the model of the feedback integral controller is split into an integrator, attached to process and two proportional compensators;

$\mathrm{R}_{3}$ : the model of the exogenous system is omitted since this element doesn't belong to the control loop;

$\mathrm{R}_{4}$ : the reference generator is considered an autonomous step generator.

Hence, the NCS model is obtained through the following steps:

$\mathrm{S}_{1}$ : the state space model (5), of state variable $\mathrm{x}_{\mathrm{i}}$, is associated to the integrator from (3):

$$
\left\{\begin{array}{l}
x_{i}[k+1]=x_{1}[k]+u_{i}[k] \\
u_{c}[k]=x_{i}[k]+u_{i}[k]
\end{array},\right.
$$

where $\mathrm{u}_{\mathrm{i}}$ is the output of the controller corresponding to the gain $\mathrm{K}_{\mathrm{i}}$;

$\mathrm{S}_{2}$ : the first order linear model (6), of state variable $\mathrm{X}_{\mathrm{w}}$, is introduced for the reference generator:

$$
\left\{\begin{array}{l}
x_{w}[k+1]=x_{w}[k] \\
w[k]=x_{w}[k]
\end{array}\right.
$$

$\mathrm{S}_{3}$ : the extended state space process model (7) of the process, observer, integrator and reference generator is obtained from equations (2) and (4) - (6), by defining the aggregated state vector

$$
x_{e}[k]=\left[\hat{x}^{T}[k] x^{T}[k] x_{i}[k] x_{w}[k]\right]^{T}
$$

and considering the connections that appear in (3):

$$
\begin{aligned}
& x_{e}[k+1]=F_{e} x_{e}[k]+G_{e} y_{N}[k] \\
& F_{e}=\left[\begin{array}{cccc}
F_{d}-\mathbf{L} \mathbf{H}_{d}^{T} & \mathbf{L} \mathbf{H}_{d}^{T} & G_{d} & G_{d} K_{i} \\
0_{n_{p} \times n_{p}} & F_{d} & G_{d} & G_{d} K_{i} \\
0_{1 \times n_{p}} & 0_{1 \times n_{p}} & 1 & K_{i} \\
0_{1 \times n_{p}} & 0_{1 \times n_{p}} & 0 & 1
\end{array}\right] \\
& G_{e}=\left[\begin{array}{c}
-G_{d} K_{1} \\
-G_{d} K_{1} \\
K_{2} \\
0_{1 \times n}
\end{array}\right] \text {, with } \mathbf{K}_{1}=\left[\mathbf{K K}_{\mathrm{i}}\right], \mathbf{K}_{2}=\left[0 \mathrm{~K}_{\mathrm{i}}\right]
\end{aligned}
$$

$\mathrm{S}_{4}$ : the extended process model is further coupled with the network model (1) through the state aggregation

$$
\bar{x}_{N}[k]=\left[\tilde{x}_{N}^{T}[k] x_{e}^{T}[k]\right]^{T}
$$

resulting the closed loop autonomous switched linear system:

$$
\bar{x}_{N}[k+1]=\bar{A}_{\alpha}[k], \delta[k] \bar{x}_{N}[k]
$$

where

$$
\begin{aligned}
& \bar{A}_{\alpha[k], \delta[k]}=\left[\begin{array}{ll}
A_{\alpha[k], \delta[k]} & B_{\alpha[k]} B_{1} \\
G_{e, \alpha[k]} C & F_{e, \alpha[k]}
\end{array}\right] \\
& B_{1}=\left[\begin{array}{llllll}
I_{n_{p}} & H_{d}^{T} & 0 & 0 & 0 & 0
\end{array}\right]
\end{aligned}
$$

The system (8) is a time-variant system whose dynamic at any time instant $\mathrm{k}$ depends on the switching signal pair $(\alpha, \delta) \in \Psi=\Omega_{1} \times \Omega_{2}$. Let $(\alpha, \delta)=(i, j)$ at moment $k$ and $(\alpha, \delta)=(u, v)$ at the consecutive sampling instance $k+1$. Then, the following theorem gives a sufficient condition for asymptotic stability for the closed loop model of the NCS (8):

Theorem 1. The autonomous switched linear system (8) is asymptotically stable if along each state trajectory, point by point, there exists positive definite symmetric matrices $\left\{\mathbf{P}_{\mathrm{i}, \mathrm{j}} \mid(\mathrm{i}, \mathrm{j})\right.$ $\in \Psi\}$, such that

$$
\begin{aligned}
& \bar{A}_{i, j}^{T} P_{u, v} \bar{A}_{i, j}-P_{i, j}<0, \\
& \forall((i, j),(u, v)) \in \Psi \times \Psi
\end{aligned}
$$

The proof of the theorem, based on a switched quadratic Lyapunov candidate function of the form:

$$
V[k]=\bar{x}_{N}^{T}[k] P_{a[k], \delta[k]} \bar{x}_{N}[k]
$$

can be found in [7] for a switched linear system with a single switching signal, and in [22] for systems with two switching signals like (8).

In principle, the application of Theorem 1 requires to investigate the existence of positive definite symmetric matrices $\left\{\mathbf{P}_{\mathrm{i}, \mathrm{j}} \mid(\mathrm{i}, \mathrm{j}) \in \Psi\right\}$ that verify (9) for any arbitrary switching of the signals pair $(\alpha, \delta) \in \Psi$ between two consecutive moments.

Now, by using the Schur complement formula, condition (9) of Theorem 1 can be replaced by the following linear matrix inequality (LMI) problem:

$$
\left[\begin{array}{cc}
P_{i, j} & \bar{A}_{i, j}^{T} P_{u, v} \\
P_{u, v} \bar{A}_{i, j} & P_{u, v}
\end{array}\right]>0,
$$


that can be numerically solved by formulating the problem as a convex optimization one ([3]). Consequently, instead of Theorem 1, the following corollary can be applied:

Corollary 1. The autonomous switched linear system (8) is asymptotically stable if along each state trajectory, point by point, there exists symmetric positive definite matrices $\left\{\mathbf{P}_{\mathrm{i}, \mathrm{j}} \mid(\mathrm{i}, \mathrm{j})\right.$ $\in \Psi\}$, such that the LMI conditions from (11) are simultaneously fulfilled.

\section{Case Study}

A permanent magnet $\mathrm{DC}$ motor of $20 \mathrm{~W}$ is considered as the controlled process in Figure 2. The motor has a moment of inertia $\mathbf{J}=$ $5.18 \cdot 10^{-6} \mathrm{~kg} \mathrm{~m}^{2}$, and a maximum speed $\mathrm{y}_{\max }=$ $4000 \mathrm{rpm}$. The control variable value ranges between $\pm 30 \mathrm{~V}$. Next, a second order continuous time model, established by imposing a constant load torque and by omitting frictions in bearings, is considered for the motor. The parameters of the DC motor were identified experimentally. As a final result, for the linearized model around the equilibrium point defined by $\mathrm{y}_{\mathrm{o}}=1268 \mathrm{rpm}, \mathrm{u}_{\mathrm{o}}=2.6 \mathrm{~V}$ and a constant load $(\approx 1 / 4$ of the nominal load), the zero-order hold equivalent corresponding to a sample rate $h=1 \mathrm{~ms}$ is obtained (12).

$$
\left\{\begin{array}{l}
{\left[\begin{array}{l}
x_{1}[k+1] \\
x_{2}[k+1]
\end{array}\right]=\overbrace{\left[\begin{array}{rr}
0.99 & 0.06 \\
-0.23 & 0.77
\end{array}\right]}^{F_{b}} \overbrace{\left[\begin{array}{l}
x_{1}[k] \\
x_{2}[k]
\end{array}\right]}^{F^{[k]}}+\overbrace{\left[\begin{array}{l}
0.01 \\
0.23
\end{array}\right]}^{G_{d}}} \\
y[k]=\overbrace{\left[\begin{array}{ll}
1 & 0
\end{array}\right]\left[\begin{array}{l}
x_{1}[k] \\
x_{2}[k]
\end{array}\right]}
\end{array}\right.
$$

In (12) $x_{1}=y$ represents the variation of the motor's speed in respect to $y_{0}, x_{2}$ the armature current variation, and $\mathrm{u}$ the variation of the control variable in respect to $u_{o}$ given by the control law (3).

The communication network, described by model (1), is characterized by $\tau_{\max }=7$, which leads to $\Omega_{1}=\{1,2,3,4,5,6,7\}$. The scenario corresponds to a packet switched local area communication network (LAN) with a maximum round trip time (RTT) of $6 \mathrm{~ms}$ (the RTT represents the combined one way delay (OWD) of both ways of the network).

The controller (3) and the observer (4) were designed according to the previous section, by allocating the poles like in Figure 4. The positioning of the observer's poles in respect to those of the control loop (without the observer) assures a faster observation dynamic than that of the control loop. Finally the following gains were obtained: $\mathrm{K}_{\mathrm{i}}=22.12, \mathbf{K}=\left[\begin{array}{ll}3.20 & 0.79\end{array}\right]$.

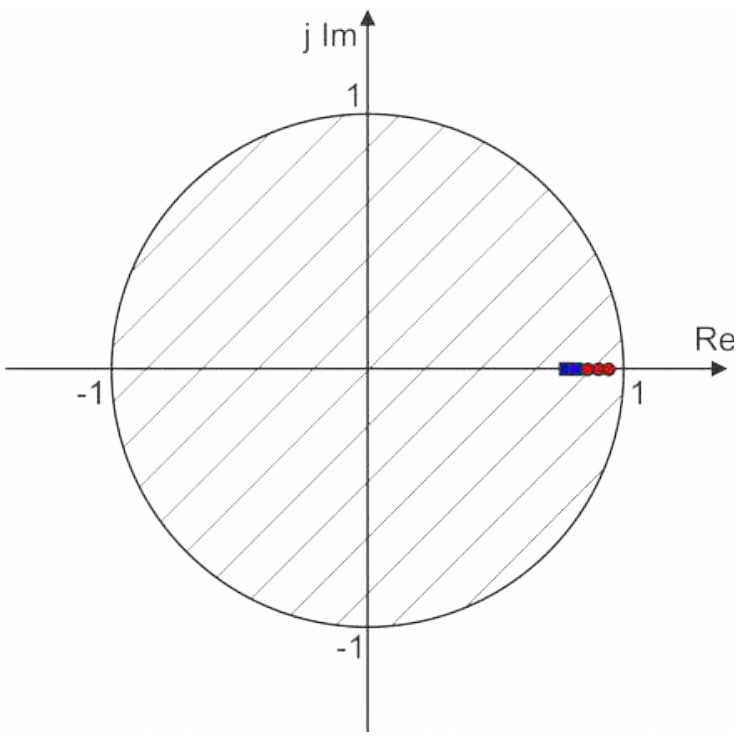

Figure 4. Poles placement diagram: $\mathrm{p}_{1}=0.85, \mathrm{p}_{2}=$ $0.84, \mathrm{p}_{3}=0.87-$ control system's poles (red); $\mathrm{p}_{\mathrm{o} 1}=$ $0.83, \mathrm{p}_{\mathrm{o} 2}=0.81-$ observer's poles (blue).

The next design step consists in the stability assessment of the whole system (8). The problem is reduced to proofing the existence of a set of $\mathbf{P}_{\mathrm{i}, \mathrm{j}}$ matrices fulfilling the conditions (11). For this, the multiple LMI conditions (11) are recasted as a single LMI, which is solved using the CVX Toolbox for Matlab ([10]), by formulating the problem as a convex optimization one. A solution composed of a set of $14 \mathbf{P}_{\mathrm{i}, \mathrm{j}}$ matrices was obtained. Hence, according to Corollary 1, the system (8) is stable. In other words, the trajectories generated by the set of all switching signals pair $(\alpha, \delta)$ of the control system show a stable behaviour.

Next, in order to illustrate the behaviour of the previously designed control system, a simulation scenario is presented. The scenario considers an overloaded multi-hop switched Ethernet LAN. The implementation structure, based on the NCS from Figure 3, is depicted in Figure 5.

For the Network block, the Network Emulator described in [23] was implemented. The logic diagram of the Network Emulator's algorithm is presented in Figure 6. Here, $\mu, \tau$ and $p$ are the input signals ( $\tau$ and $\mathrm{p}$ have the same meaning as in Section 2), $\eta$ is the output signal and $\varphi$ is the states vector.

The $\tau$ and $p$ signals are obtained using the NS-2 network simulation software ([19]). The 


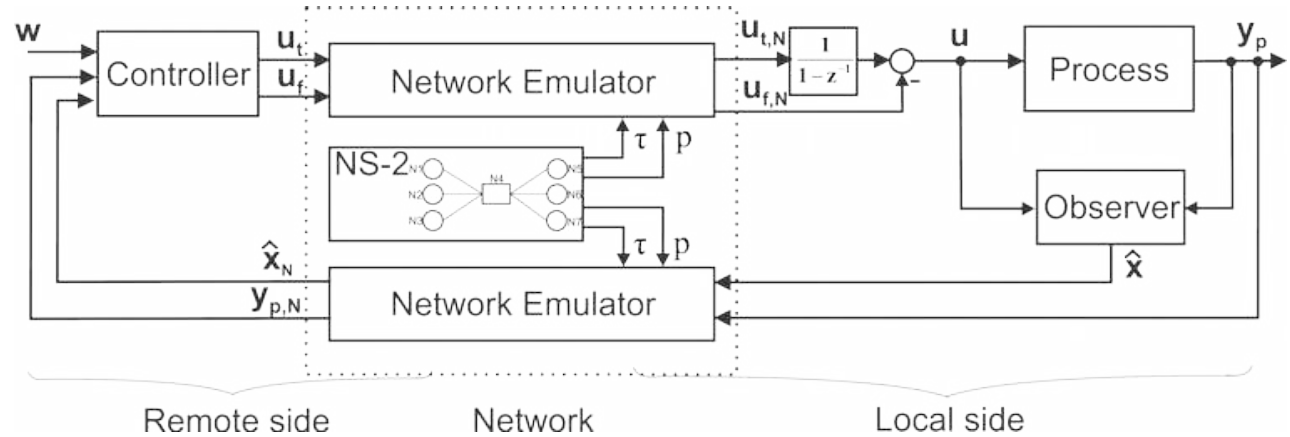

Figure 5. NSIS-based networked control system - implementation structure

scenario conducts a comparative study between two cases. In the first case, named S-SE, (Figure 7), the network nodes communicate via standard Switched Ethernet connections, while in the second case, named NSIS-SE, (Figure 8), the network nodes implement the NSIS protocol suite for Switched Ethernet using a modified version of the NS-2 module proposed in [4].

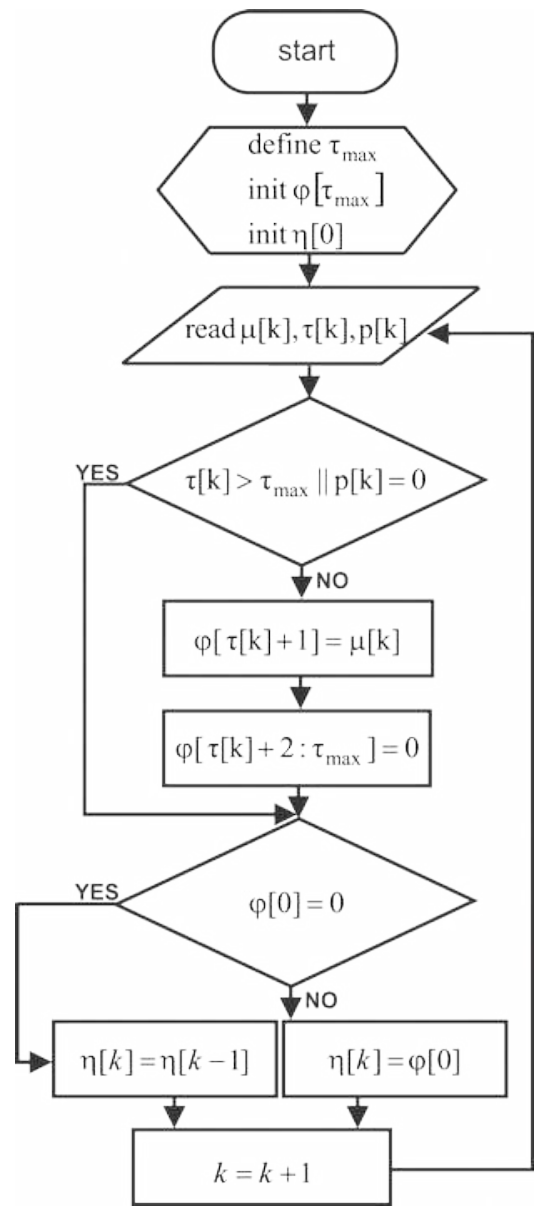

Figure 6. Logic diagram of the Network Emulator's algorithm

The setup implemented in the NS-2 simulation software considers a LAN composed of seven network nodes (see Figure 5), having a maximum imposed end-to-end propagation delay of $0.3 \mathrm{~ms}$. Throughout the entire simulation, two file transfer protocol (FTP) data streams generate network traffic covering $50 \%$ of the network throughput capacity. At moment $\mathrm{t}=0$ of the simulation, two additional user datagram protocol (UDP) data flows start. The first data flow considered, $\mathrm{DF}_{1}$, transmits Ethernet packets at a constant data rate (one packet of 1500 bytes maximum transmission unit (MTU) every millisecond). The second data flow, $\mathrm{DF}_{2}$, is a generic transport layer data flow, obtained from an exponential traffic source, capable of generating random data at a maximum rate of $60 \%$ of the network throughput capacity. $\mathrm{DF}_{1}$ simulates the network transport of the NCS's digital signals, while $\mathrm{DF}_{2}$ simulates a disturbance data flow capable of network congestion, when combined with the FTP streams.

Figure 7 a) and Figure 8 a) illustrate, for the considered cases, the time varying delay associated with each transport layer data flow $\left(\mathrm{DF}_{1}\right.$ - yellow, $\mathrm{DF}_{2}$ - red). As one can see, when using the NSIS protocol suite, the maximum OWD of the $\mathrm{DF}_{1}$ traffic (NCS's signals transport) do not exceed $3 \mathrm{~ms}\left(\tau_{\max }=3\right)$, as opposed to the standard setup when the OWD has a maximum value of $14 \mathrm{~ms}\left(\tau_{\max }=\right.$ 14). In both cases, because the Ethernet Switch won't reach the saturated state, the packet loss rate is negligible.

The same NS-2 simulation results ( $\tau$ and $p$ signals associated to the $\mathrm{DF}_{1}$ flow) are considered as inputs of both Network Emulators on the direct and the feedback path of the NCS from Figure 5, by taking into account a symmetric traffic distribution on both ways of the network.

The initial conditions for the plant states are null. A step reference signal is adopted as $\mathrm{w}[\mathrm{k}]$ $=10 \sigma[\mathrm{k}] \mathrm{rad} / \mathrm{s}(300 / \pi \mathrm{rpm})$. Figure $7 \mathrm{~b})$ shows 
the response of the NCS, when using the standard switched Ethernet network. Here, the system becomes unstable due to the network disturbance. Figure $8 \mathrm{~b}$ ) shows the response of the NCS when the network implements the NSIS protocol suite. The response is characterized by a settling time of $0.45 \mathrm{~s}$, a null steady state error and no overshoot.

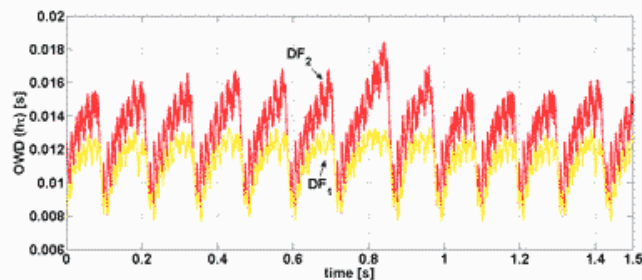

a)

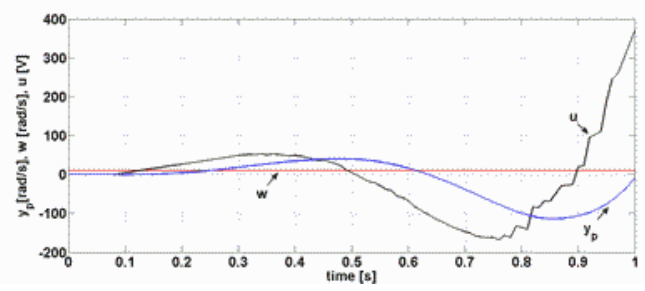

b)

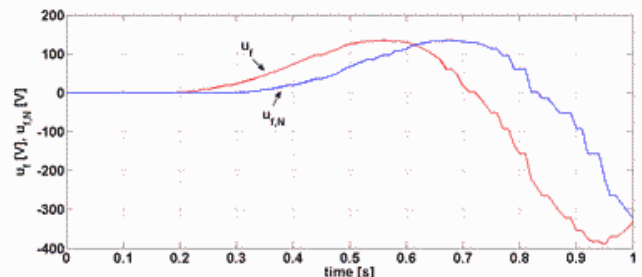

c)

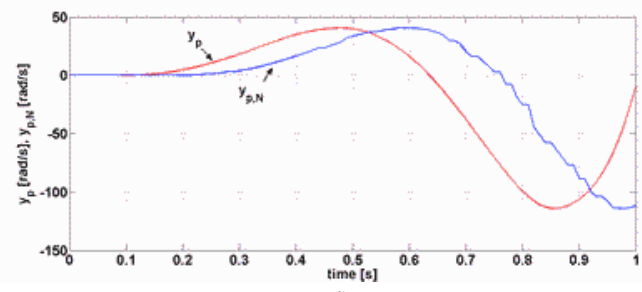

d)

Figure 7. Simulation results - case S-SE

Figure $7 \mathrm{c}$ ), d) and Figure $8 \mathrm{c}$ ), d) show the disturbance effect of the network on the NCS signals. If the control signals distortions (time shifts and deformations) are not kept by the QoS adapter in certain limits, imposed at the analysis stage of the control system, they could lead to performance degradation and ultimately to the instability of the control system (Figure 7). Figure 8 shows that by imposing a $3 \mathrm{~ms}$ limit to the time varying delay of the control signals on both the direct and the feedback path and by avoiding equipment saturation (information loss), the control system manage to compensate the disturbance effect of the network maintaining system's stability and performance.

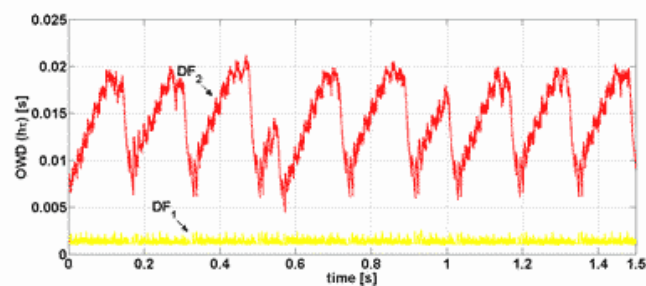

a)

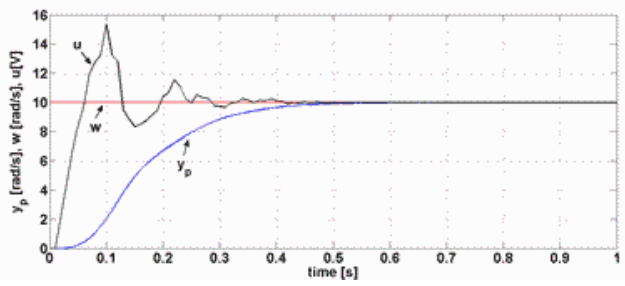

b)

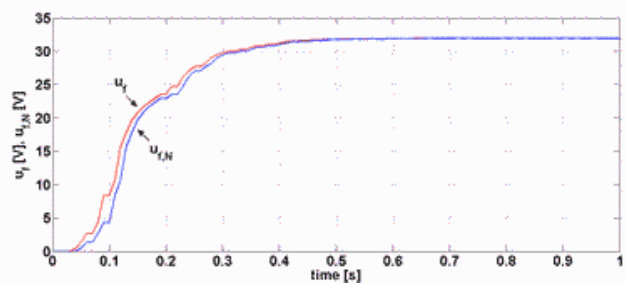

c)

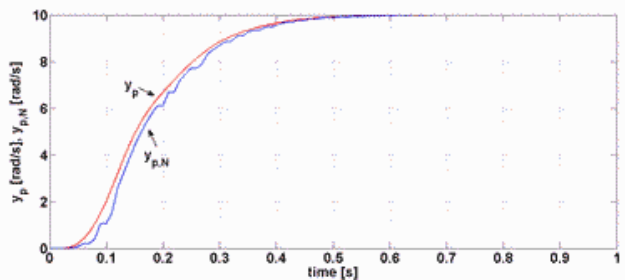

d)

Figure 8. Simulation results - case NSIS-SE

\section{Conclusions}

The article presents the modelling, design and stability analysis of a NCS based on a controlaware QoS adaptation co-design method. The NCS, composed out of a local plant, a remote controller and a network adaptation block, continuously adjust the network parameters in order to meet the control objectives. A novel QoS adapter design is proposed, using the NSIS protocol suite for end-to-end QoS. The system's stability is analysed by considering the overall system as an autonomous switched linear one with multiple switching signals. Finally, a case study illustrates through simulations the effectiveness of the control solution.

\section{Acknowledgements}

This work was partially supported by the strategic grant POSDRU/159/1.5/S/137070 (2014) of the Ministry of National Education, Romania, co-financed by the European Social 
Fund - Investing in People, within the Sectoral Operational Programme Human Resources Development 2007-2013.

\section{REFERENCES}

1. AUBRUN, C., D. SIMON, Y.-Q. SONG, Ed., Co-design Approaches for Dependable Networked Control Systems, Wiley, 2010.

2. BAI, J., E. P. ELISI, F. QIU, Y. XUE, X. D. KOUTSOUKOS, Optimal Cross-Layer Design of Sampling Rate Adaptation and Network Scheduling for Wireless Networked Control Systems, IEEE/ACM Third International Conference on CyberPhysical Systems (ICCPS), Beijing, 2012, pp. 107-116.

3. BOYD, S., L. EL GHAOUI, E. FERON, V. BALAKRISHNAN, Linear Matrix Inequalities in System and Control Theory, SIAM, 1994.

4. CARMO, M., B. CARVALHO, J. S. SILVA, E. MONTEIRO, P. SIMÕES, M. CURADO, F. BOAVIDA, NSIS-based Quality of Service and Resource Allocation in Ethernet Networks, Proceedings of the 4th International Conference on Wired/Wireless Internet Communications, Bern, 2006.

5. CARUNTU, C. F., C. LAZAR, Networked Predictive Control for Timevarying Delay Compensation with an Application to Automotive Mechatronic Systems, Journal of Control Engineering and Applied Informatics, vol. 13, no. 4, 2011, pp. 19-25.

6. CLOOSTERMAN, M. B. G., L. HETEL, N. VAN DE WOUW, W. P. M. H. HEEMELS, J. DAAFOUZ, H. NIJMEIJER, Controller Synthesis for Networked Control Systems, Automatica, vol. 46, 2010, pp. 1584-1594.

7. DAAFOUZ, J., P. RIEDINGER, C. IUNG, Stability Analysis and Control Synthesis for Switched Systems, IEEE Transactions on Automatic Control, vol. 47, no. 11, 2002, pp. 1883-1887.

8. DE MORAES, R. A. R, F. VASQUES, A Quality-of-Service (QoS) Based Approach for the Communication Support in Network-based Control Systems: an On- going Project, $11^{\text {th }}$ IFAC Symposium on Information Control Problems in Manufacturing, Salvador, 2004.

9. DIOURI, I., J. GEORGES, E. RONDEAU, Accommodation of Delays for NCS using Classification of Service, International Conference on Networking, Sensing and Control, London, 2007, pp. 410-415.

10. GRANT, M., S. BOYD, CVX: Matlab Software for Disciplined Convex Programming, version 1.21., 2011, http://cvxr.com/cvx.

11. HESPANHA, J. P., P. NAGHSHTABRIZI, $Y$. XU, A survey of Recent Results in Networked Control Systems, Proceedings IEEE, vol. 95, no. 1, 2007, pp. 138-162.

12. HU, W., G. LIU, D. REES, Event-Driven Network Predictive Control, IEEE Transactions on Industrial Electronics, vol. 59, no. 3, 2011, pp. 905-913.

13. KAPPlER, C., X. FU, B. SCHLOER, A QoS Model for Signaling IntServ Controlled-Load Service with NSIS, Internet draft, work in progress, 2011, https://tools.ietf.org/html/draft-kapplernsis-qosmodel-controlledload-14.

14. LAI, C.-L., P.-L. HSU, Design the Remote Control System With the Time-Delay Estimator and the Adaptive Smith Predictor, IEEE Transactions on Industrial Informatics, vol. 6, no. 1, 2010, pp. 73-80.

15. LI, H., Z. SUN, M.-Y. CHOW, F. SUN, Gain-Scheduling-Based State Feedback Integral Control of Networked Control Systems, IEEE Transactions on Industrial Electronics, vol. 58, no. 6, 2011, pp. 2465-2472.

16. LUENBERGER, D. G., An Introduction to Observers, IEEE Trans. on Automatic Control, vol. 16, no. 6, 1971, pp. 596-602.

17. NATORI, K., T. TSUJI, K. OHNISHI, A. HACE, K. JEZERNIK, Time-Delay Compensation by Communication Disturbance Observer for Bilateral Teleoperation under Time-varying Delay, IEEE Transactions on Industrial Electronics, vol. 57, no. 3, 2010, pp. 1050-1062.

18. NILSSON, J., Real-time Control Systems with Delay, Ph.D. Thesis, Lund Institute of Technology, Lund, 1998. 
19. NS-2 network simulation software, 2014, http://www.isi.edu/nsnam/ns/.

20. SOGA, T., N. OTSUKA, Stabilizability Conditions for Switched Linear Systems with Constant Input via Switched Observer, Studies in Informatics and Control, vol. 22, no. 1, 2013, pp. 7-14.

21. SONG, Y.-Q., Networked Control Systems: From Independent Designs of the Network QoS and the Control to the Co-design, 8th IFAC International Conference on Fieldbuses and Networks in Industrial and Embedded Systems, Ansan, 2009, pp. 155-162.

22. STEFAN, O., A. CODREAN, T.-L. DRAGOMIR, A Network Control Structure with a Switched PD Delay Compensator and a Nonlinear Network Model, American Control Conference, Washington, 2013, pp. 758-764.
23. STEFAN, O., A. CODREAN, T.-L. DRAGOMIR, A Nonlinear State Space Model of Network Transmissions in a Network Control System, Journal of Control Engineering and Applied Informatics, vol. 13, no. 4, 2011, pp. 58-63.

24. TIPSUWAN, Y., M. Y. CHOW, Control Methodologies in Networked Control Systems, Control Engineering Practice, vol. 11, 2003, pp. 1099-1111.

25. XIAOMING, F., H. SCHULZRINNE, A. BADER, D. HOGREFE, C. KAPPLER, G. KARAGIANNIS, H. TSCHOFENIG, S. VAN DEN BOSCH, NSIS: A New Extensible IP Signaling Protocol Suite, IEEE Communications Magazine, vol. 43, no. 10,2005 , pp. 133-141. 